\title{
Global-phase seismic interferometry unveils P-wave reflectivity below the Himalayas and Tibet
}

\author{
Elmer Ruigrok $^{1}$ and Kees Wapenaar ${ }^{1}$ \\ Received 12 March 2012; revised 24 April 2012; accepted 30 April 2012; published 5 June 2012.
}

[1] A number of seismic methods exist to image the lithosphere below a collection of receivers, using distant earthquakes. In the current practice, especially mode-conversions in teleseismic phases are utilized. We present a new method that takes advantage of the availability of global phases. This method is called global-phase seismic interferometry (GloPSI). With GloPSI, zero-offset reflections are extracted from reverberations near the array caused by global seismicity. We exemplify GloPSI with data from the Hi-CLIMB experiment (2002-2005) and migrate the obtained reflection responses. This results in a $800 \mathrm{~km}$ long reflectivity profile through the Himalayas and a large part of the Tibetan Plateau. Citation: Ruigrok, E., and K. Wapenaar (2012), Global-phase seismic interferometry unveils $\mathrm{P}$-wave reflectivity below the Himalayas and Tibet, Geophys. Res. Lett., 39, L11303, doi:10.1029/2012GL051672.

\section{Introduction}

[2] A number of seismic methods exist to image the lithosphere below a collection of receivers, using distant earthquakes. Although both global phases (i.e., waves that traverse the core before reaching a receiver) and teleseismic phases (i.e., waves that travel through the mantle, but not through the core, before reaching a receiver) could be used, in the current practice, especially teleseismic phases are utilized. In this paper we present a method that takes advantage of the availability of global phases.

[3] Claerbout [1968] showed that the reflection response of a horizontally layered acoustic medium can be retrieved from the autocorrelation of a plane-wave transmission response. Later, his theory was generalized to arbitrarily heterogeneous (and elastic) media [Wapenaar, 2003; Derode et al., 2003; Schuster et al., 2004]. Hence, nowadays we know how to retrieve $\mathrm{P}$ - and $\mathrm{S}$-wave reflection responses between any two receivers at the Earth's surface. However, a wide distribution of earthquakes is required to achieve this [Abe et al., 2007; Ruigrok et al., 2008, 2010]. This distribution does not always exist or it might take ages to detect. In this paper we propose a simple, but effective, alternative. We turn back to the original setting from Claerbout's paper and translate it to global-scale seismology. This new adaption we call Global-Phase Seismic Interferometry, which is abbreviated as GloPSI.

\footnotetext{
${ }^{1}$ Department of Geoscience and Engineering, Delft University of Technology, Delft, Netherlands.

Corresponding author: E. Ruigrok, Department of Geoscience and Engineering, Delft University of Technology, Stevinweg 1, 2628 CN Delft, Netherlands. (e.n.ruigrok@tudelft.nl)

Copyright 2012 by the American Geophysical Union. 0094-8276/12/2012GL051672
}

[4] GloPSI can be applied on a single seismic station installed on a lithosphere of investigation. The P-wave reflection response is extracted by autocorrelating phaseresponses. Considering the lithosphere is - grosso modohorizontally layered, only incoming waves which impinge on the lithosphere near vertical incidence are needed. Thus, global phases, like PKIKP, are used. These global phases may be induced over a large area at the other side of the globe. Due to the small angles of incidence, the source Fresnel zones for global phases are large. Consequently, the large area for which global phases exist only needs to be sparsely populated with seismicity. Another advantage of global phases is that the vertical-component record is already a good approximation of the P-wave transmission response. Consequently, the lower signal-to-noise of the horizontal components can be left out of the equation. For an array of receivers, the extracted reflection responses can be used to image the P-wave reflectivity of the lithosphere.

[5] In the following we further explain GloPSI and apply it to data from the Hi-CLIMB experiment (2002-2005). This was a large and well-sampled seismic array, passing the Himalayas and a significant part of the Tibetan Plateau. For this array, images have been published, which were obtained by applying a wide suite of different geophysical techniques [Chen et al., 2010; Griffin et al., 2011; Hung et al., 2011; Nábělek et al., 2009; Nowack et al., 2010; Tseng et al., 2009; Xu et al., 2011]. This enables benchmarking of the GloPSI results.

\section{Global-Phase Seismic Interferometry}

[6] Body waves from earthquakes at global distances travel through the mantle and core before they reach an array of seismic stations. Different stationary raypaths exist. Most prominent are $P K P, P K i K P$ and $P K I K P$, see Figure 1a. When these waves reach the upper mantle below the array (Figure $1 \mathrm{~b}$ ) they are nearly planar. Direct waves are followed by reverberations in the lithosphere. The reverberations that hit one station, then (multiply) reflect on subsurface interfaces before reaching a receiver again, can be isolated with a technique called seismic interferometry. In the current application we only isolate reflections received by one and the same station $\mathbf{x}_{\mathcal{A}}$, see Figure 1c. For a perfectly horizontally layered subsurface, illuminated with a plane wave, we could use the following relation [Claerbout, 1968; Wapenaar, 2003]:

$$
T\left(\mathbf{x}_{\mathcal{A}}, \mathbf{p},-t\right) * T\left(\mathbf{x}_{\mathcal{A}}, \mathbf{p}, t\right)=-R\left(\mathbf{x}_{\mathcal{A}}, \mathbf{p},-t\right)+\delta(t)-R\left(\mathbf{x}_{\mathcal{A}}, \mathbf{p}, t\right),
$$

where $\mathrm{T}\left(\mathbf{x}_{\mathcal{A}}, \mathbf{p}, t\right)$ is the transmission response with $\mathbf{p}=$ $(p, \theta) p$ is the absolute horizontal rayparameter, $\theta$ is the backazimuth, $R\left(\mathbf{x}_{\mathcal{A}}, \mathbf{p},-t\right)$ is the plane-wave reflection 

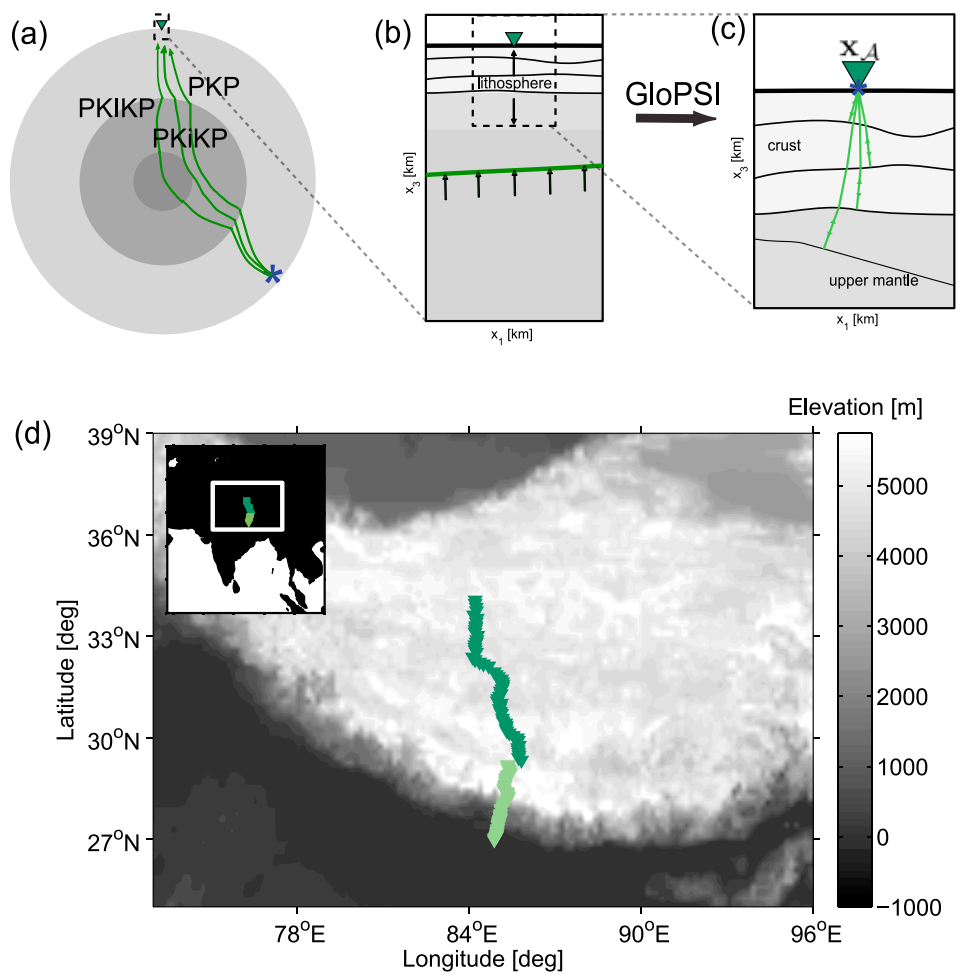

Figure 1. (a) Raypaths (green lines) between a source (blue star) and a receiver (green triangle) for the most prominent global phases. (b) Enlargement of the medium below the receiver, with a global phase wavefront (green line) just before it impinges on the lithosphere. (c) The result of applying Global-Phase seismic interferometry (GloPSI), which is the zero-offset reflection response. (d) Layout of the 2 selected subarrays from Hi-CLIMB, the Himalaya array (light-green triangles) and the Tibet array (dark-green triangles) plotted on top of an elevation map of the Tibetan Plateau.

response, $\delta(t)$ is a delta pulse and * denotes convolution. Thus the reflection response is obtained by autocorrelating the transmission response, selecting minus the causal result and muting the delta pulse.

[7] In reality the lithosphere is not perfectly horizontally layered. Moreover, transmission responses are colored with source time functions $s_{i}(t)$, where $i$ is a source index. Therefore we extend equation (1) to

$$
\begin{aligned}
& \sum_{\theta_{\min }}^{\theta_{\max }} \sum_{p_{\min }}^{p_{\max }} T\left(\mathbf{x}_{\mathcal{A}}, \mathbf{p},-t\right) * s_{i}(-t) * T\left(\mathbf{x}_{\mathcal{A}}, \mathbf{p}, t\right) * s_{i}(t) \\
& \quad \propto\left\{-R\left(\mathbf{x}_{\mathcal{A}}, \mathbf{x}_{\mathcal{A}},-t\right)+\delta(t)-R\left(\mathbf{x}_{\mathcal{A}}, \mathbf{x}_{\mathcal{A}}, t\right)\right\} * S_{n}(t),
\end{aligned}
$$

where $S_{n}(t)$ is the average of autocorrelations of the different source time functions. Thus, we repeat the autocorrelation for plane waves with varying illumination and stack the results. Through the autocorrelation, both physical and spurious events are created. By stacking the correlations over an adequate illumination range, the stationary events are enhanced [Snieder, 2004] while the spurious events, amongst others cross terms due to heterogeneities near the earthquake sources [Ruigrok et al., 2010], are suppressed. For global phases the rayparameter varies from $p_{\min }=0$ to $p_{\max }=$ $0.04 \mathrm{~s} / \mathrm{km}$. The backazimuth may range from $\theta_{\min }=0$ to $\theta_{\max }=360^{\circ}$. This illumination range suffices to retrieve the zero-offset (the zero-offset response is the response that would be measured if there were a coinciding source and receiver at the free surface) reflection response $R\left(\mathbf{x}_{\mathcal{A}}, \mathbf{x}_{\mathcal{A}}, t\right)$ from horizontal and gently dipping interfaces. Nowack et al. [2006] applied autocorrelation, prior to migration, to only a single teleseismic phase and showed that still an acceptable image could be obtained in a setting with steeply dipping structure.

\section{Data Selection}

[8] We exemplify GloPSI with data from the Hi-CLIMB (Himalayan-Tibetan Continental Lithosphere During Mountain Building) experiment (2002-2005) [e.g., Nábělek et al., 2009; Nowack et al., 2010]. A large array of stations was laid out to image a transect of the Indian-Eurasian Plate collision. Over the years, the array had a station distribution moving up from Northern India to Nepal and China. We select two approximately linear subarrays. Figure 1d depicts the setting of the Himalaya subarray (58 stations, denoted with darkgreen triangles) and Tibet subarray (61 stations, denoted with light-green triangles). The two subarrays were not simultaneously in the field. Consequently, we have two separate datasets. Below we describe the data selection for the Tibet array.

[9] Figure 2a shows the area (light-gray shading) from which direct waves to the Hi-CLIMB subarray would travel through the core. We select 25 earthquakes with $M>6$ which are located within, or just outside, this area. For each earthquake we select a time window that includes the first arrival and subsequent reverberations in the lithosphere. The first arrival is either PKiKP or PKIKP [see, e.g., Stein and 

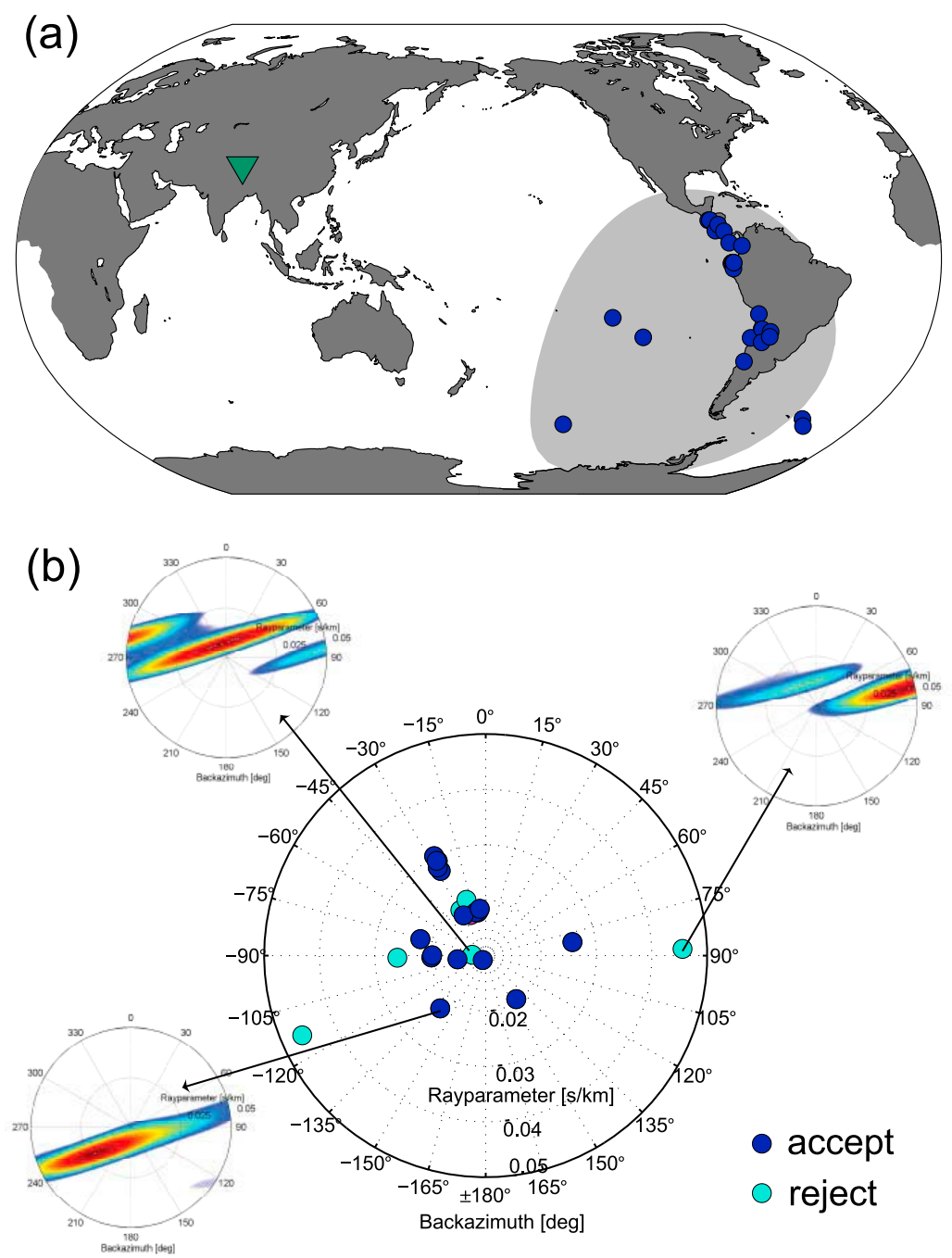

Figure 2. (a) The location of the Hi-CLIMB experiment (green triangle). The 25 selected earthquakes (blue dots) are all from within or near the area for which PKIKP exists (light-gray shading). (b) The middle figure shows the dominant (plane-wave) illumination for the 25 global-phase responses, of which 17 pass the beamforming test (accept, see main text) and 8 do not pass (reject). The dominant illumination is determined by selecting the coordinate with the largest beampower after beamforming. For the beamforming, all available Hi-CLIMB stations are used. Three examples of beampower plots are shown in the margins of Figure $2 b$.

Wysession, 2003]. The upper bound of the time-window is limited by $P P$, which is not included due to its high rayparameter. This restriction leaves time-windows lasting between 150 and $350 \mathrm{~s}$, depending on the great-circle distance.

[10] Global phase responses may be rather complex due to triplications, e.g., one selected time window may contain both a PKIKP and PKP phase response. Autocorrelating such a time window would lead to cross terms between $P K I K P$ and $P K P$, which do not contain subsurface information below the array. To limit the occurrence of such cross terms we only use the time windows with a single dominant phase response. We use beamforming [e.g., Rost and Thomas, 2002] (see Figure 2b) to study the effective illumination. Hence, we reject time windows which are not dominated by a single rayparameter. Also, we reject time windows which are dominated by an incoming wave with a significant angle of incidence (a rayparameter exceeding
$0.04 \mathrm{~s} / \mathrm{km}$ ). Consequently, 17 phase responses remain for further processing.

[11] For the Himalaya array there are less global phases available induced by $M>6$ earthquakes. Therefore, we lower the threshold to $M>5.5$ and find 52 global phases. Using the same procedure as described above, 34 phases remain for further processing.

\section{Processing}

[12] We use the Z-component records as approximations of the P-wave transmission responses. To all selected phase responses we apply instrument-response deconvolution and bandpass filtering ([0.01 2.0] Hz). Subsequently, we apply equation (2). Thus, e.g., for the southernmost station in the Tibet (or Himalaya) array, the phase response of the first earthquake is autocorrelated. To this autocorrelation, the autocorrelation of the other 16 (or 33 for the Himalaya array) phase responses are added and the causal times are selected. 

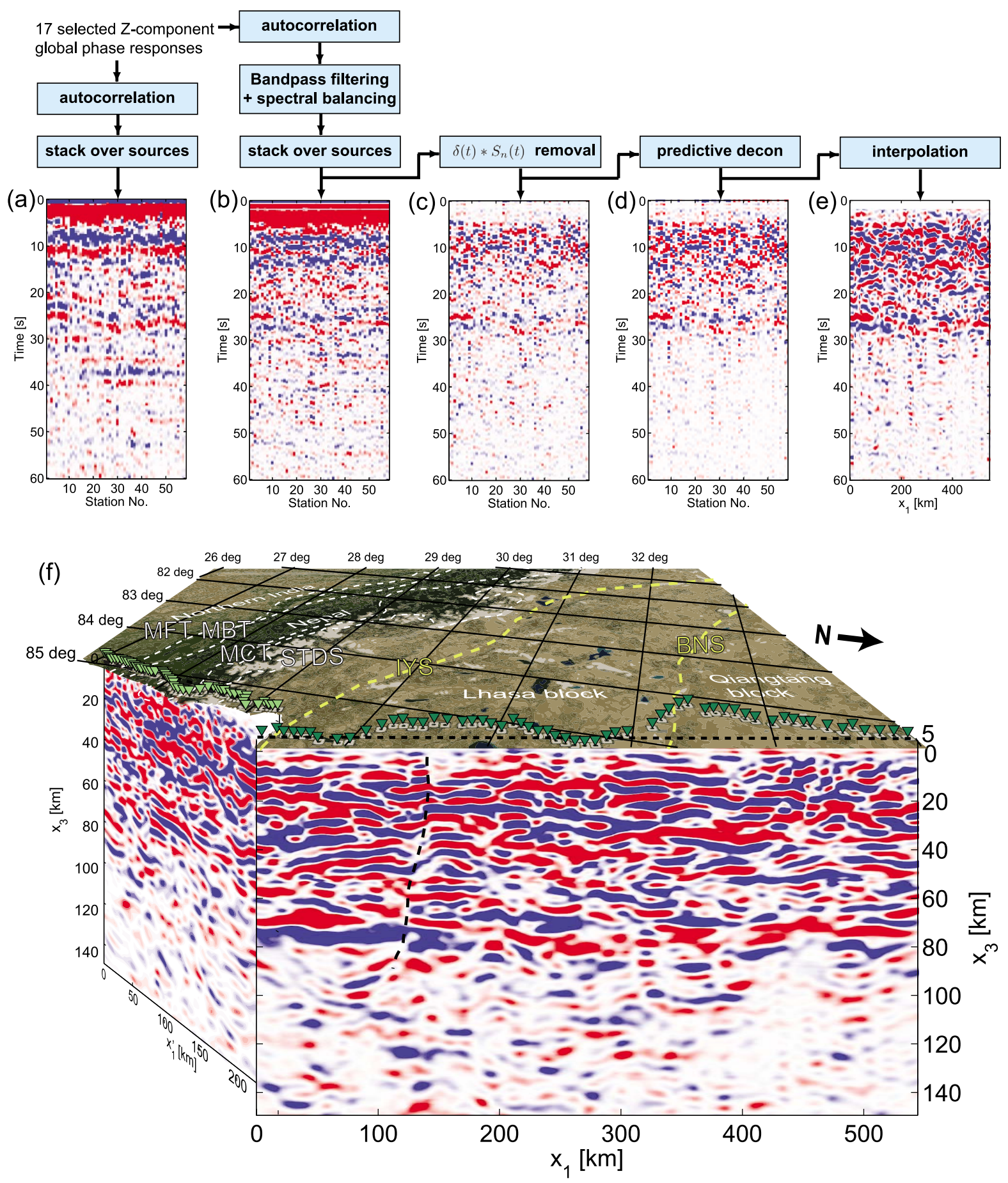

Figure 3. (a-e) Five stages of the estimated zero-offset reflection response below the Tibet array (Figure 1d). From left to right, the estimation of the primary reflection response is improved by a succession of pre- and postprocessing algorithms (see text). The used algorithms are indicated on top of the zero-offset panels, in the form of a flowchart. (f) The P-wave reflectivity images for (left) the Himalaya array and (right) the Tibet array, positioned below a satellite image of the Central and Western Tibetan Plateau. (For a non-perspective view of the Himalaya-array results, see Figure S3.) On the left-hand side, the snow-covered (i.e., white) Himalayas can be seen rising from the lush (i.e., green) Indo-Gangetic Plains to the plateau. The main tectonic blocks are indicted as well as the zones separating them. Left are the major shear zones related to the formation of the Himalayas (dashed white lines): MFT (Main Frontal Thrust), MBT (Main Boundary Thrust), MCT (Main Central Thrust) and STDS (South Tibetan Detachment System). Right are the suture zones related to the formation of the Tibetan Plateau (dashed yellow lines): IYS (Indus Yarlung Suture) and BNS (Banggong Nujiang Suture) [e.g., Yin and Harrison, 2000]. On the Tibet reflectivity image the northern limiting fault of the Gyirong Graben (black dashed line) is indicated. On the satellite image are indicated the actual station locations (green triangles) and the lines on which the data points have been projected (dashed black lines). The depth dimension $\left(x_{3}\right)$ has been exaggerated by a factor of 2 with respect to the other dimensions. 
This gives the first trace in Figure 3a (or Figure S1a in the auxiliary material). ${ }^{1}$ The same process is repeated for all the other stations, yielding the other traces of the panel in Figure 3a (or Figure S1a). This image is already a crude estimation of the zero-offset reflection response. However, the low frequency band dominates for global-phases and hence the low resolution. Moreover, the spectral balance differs widely for the different phase responses, which impedes an optimal stacking. Therefore, we select a frequency band for which there is signal in all phase responses ([0.04 0.8] Hz) and we apply a spectral balancing [e.g., Bensen et al., 2007] before stacking, giving Figure 3b (or Figure S1b). Through this processing, both the resolution is improved and spurious events are more successfully stacked out. Still a large artifact remains around $t=0$, which is a pulse at $t=0$ convolved with the average autocorrelation of the 17 (or 34) earthquake source time functions $\left(\delta(t) * S_{n}(t)\right.$, see equation (2)). Here we can take advantage of the length of the array crossing highly heterogeneous terrain. Thus, the actual reflectivity is little coherent over the 58 (or 61) stations while the $t=0$ artifact is coherent. Consequently, we largely suppress this artifact, while only marginally affecting the reflectivity, by subtracting the scaled average trace from each individual trace, giving Figure $3 \mathrm{c}$ (or Figure S1c).

[13] The last two processing steps are a preparation for reflectivity imaging through poststack Kirchhoff time migration [e.g., Yilmaz and Doherty, 2000]. Through the application of GloPSI, the complete reflection response is retrieved, containing both primary and multiple reflections. The primary reflections can easily be migrated into a reflectivity image. Hence, from this complete response (Figure 3c or S1c) we estimate the primary reflections only, using predictive deconvolution [Verschuur, 2006]. We assume that multiple reflections are predominantly from crustal interfaces. For each trace it is evaluated whether the events at times larger than 20 seconds can be explained as a free-surface reverberation from events arriving prior to 20 seconds. Events that can be explained as such are subtracted. For predictive deconvolution no velocity model needs to be known. This gives Figure 3d (or Figure S1d). It can be seen that some later events have been removed. Due to the large topography below the Himalaya array, from sealevel to $5 \mathrm{~km}$ height (Figure S3a), an elevation correction is applied. Using an upper crustal velocity of $5.5 \mathrm{~km} / \mathrm{s}$ we apply time shifts to the data, to move all data to a virtual recording level of $5 \mathrm{~km}$ above sealevel. The migration algorithm that we use assumes a linear array of regularly spaced datapoints. Therefore, we project the snaking succession of datapoints (Figure 1d) to two straight lines. Subsequently, we bilinearly interpolate the data to a regular station spacing of $4 \mathrm{~km}$. After interpolation we obtain Figure 3e (or Figure S1e), which is the input for migration. The reflection response is now shown as function of $x_{1}$ (or $x_{1}^{\prime}$ ), which is the (great-circle) distance with respect to the southernmost station in the array.

[14] For imaging the P-wave reflectivity at the correct points in space we use a background velocity model based on a regional-scale tomography [Griffin et al., 2011]. From the average station elevation $(5 \mathrm{~km})$ to the maximum Moho depth $(75 \mathrm{~km})$ we use a linear velocity gradient from 5.5 to

${ }^{1}$ Auxiliary materials are available in the HTML. doi:10.1029/ 2012GL051672.
$6.5 \mathrm{~km} / \mathrm{s}$. For the remaining depths we use the global 1D reference model ak135 [Kennett et al., 1995] which has an upper-mantle velocity of $8.04 \mathrm{~km} / \mathrm{s}$. The same hybrid 1D velocity model is used both for the time migration and timeto-depth conversion. The result, Figure $3 \mathrm{f}$, is discussed in the next section.

\section{Results}

[15] Figure $3 f$ shows the obtained P-wave reflectivity images, positioned below a perspective view of the Central and Western Tibetan Plateau. The emphasis is on the Tibetarray results. In Figure S3, the Himalaya-array results can be studied, as well as a larger depth range for both arrays. In these reflectivity images, a blue-red-blue alternation should be interpreted as an interface with a positive (i.e., increasing with depth) impedance contrast. Below we discuss a few features imaged below the Tibet array.

[16] The first striking thing is a strong reflectivity until about $80 \mathrm{~km}$ depth and a reduced reflectivity at greater depths. At most locations, the zone of high reflectivity (the crust) is separated from the zone of less reflectivity (the upper mantle) by a strong reflector, the Moho. The Moho is imaged as a red feature at about $75 \mathrm{~km}$ depth. The remaining side lobes of the wavelet give rise to blue features at either side of the Moho. The same transition has been imaged with P-S [Nábělek et al., 2009; Nowack et al., 2010] and S-P [Xu et al., 2011] converted phases and with a single free-surface reflected S-phase [Tseng et al., 2009]. The P-wave reflectivity image shows the crustal and upper mantel structure in more detail than previous results.

[17] In the auxiliary material (Figures S2 and S3) we compare the $\mathrm{P}$-wave reflectivity images with $\mathrm{P}$-wave velocity perturbation images, as obtained by Hung et al. [2011]. The main feature at the southside of the Tibet array is a lowvelocity zone that straddles around the Indus Yarlung Suture. This zone corresponds with the Gyirong Graben, which is one of the active rifting zones over which west-east extension of the Tibetan Plateau takes place [e.g., Randey et al., 1999]. The edge of this low-velocity zone (denoted with a dashed black line in Figure 3f) is sharply imaged with a discontinuity in reflectivity.

\section{Discussion}

[18] It is needless to say that the migrated sections (Figure 3f) could still be improved. Especially, the projection of a snaking succession of datapoints to a straight line, introduces errors, which map into the migrated image. These errors could be reduced by splitting up the array in, e.g., four piecewise linear subarrays, instead of two. Furthermore, the image could be improved by replacing the $1 \mathrm{D}$ velocity model by a $2 \mathrm{D}$ model, for the migration and time-to-depth conversion. Both changes would lead to an improved positioning of the reflectivity.

[19] In the preceding we illustrated GloPSI with two arrays of stations. Note however that GloPSI is a singlestation method. Timing errors between stations are of no concern. Only for more advanced postprocessing, arrays are required. If we had only one station, the artifact around $t=0$ could not be removed using the same array-method as we used for obtaining Figure 3c. Still one would obtain a fair estimate of the zero-offset reflection response of the 
lithosphere, as in Figure 3b, if no phase responses were used with triplication time differences of the duration of two-way traveltimes through the lithosphere.

[20] GloPSI can also be applied for global S-phases like $S K S, S K K S$ and $P K S$ to obtain an S-wave reflection response, which again could be further processed into an $\mathrm{S}$-wave reflectivity image. A disadvantage of S-phases is that they are not the first to arrive and may thus be contaminated by coda from earlier phase arrivals. However, the fact that earthquakes radiate more $\mathrm{S}$-wave than $\mathrm{P}$-wave energy, might make up for this.

[21] In this study we only used global phase responses. In principle, if there is large seismicity near the array, also $P c P, S c S$ or $P c S$ could be used. Core-reflected and coretransmitted phases have typically a different bandwidth. Hence, the resolution of the eventual image could be increased by merging responses retrieved with both types of phases.

[22] GloPSI is complimentary to the receiver function method (RF) [Langston, 1979]. Both methods can be applied to any (isolated) station, given that it has been long enough in the field to have a rich recording of high-magnitude global seismicity. For RF, all 3-component data are required, whereas for GloPSI only the Z-component suffices. With $\mathrm{RF}$, receiver-side conversions are mapped to depth. These conversions are pronounced for incoming waves with a considerable angle of incidence. For this reason, especially teleseismic waves are used. GloPSI, on the other hand, uses only global-phase responses and maps reflectivity vertically below the station(s). Hence, the data input for both methods is independent. Still, the obtained images should be consistent. Thus by applying both methods, the reliability of our subsurface image will be improved.

[23] Acknowledgments. This work is supported by The Netherlands Organization for Scientific Research NWO. We would like to thank those involved with Hi-CLIMB and IRIS DMC for acquiring and providing an excellent dataset. Shu-Huei Hung made a large contribution to the auxiliary material. We much appreciate the thorough reviews by Robert Nowack and an anonymous reviewer.

[24] The Editor thanks Robert Nowack and an anonymous reviewer for assisting with the evaluation of this paper.

\section{References}

Abe, S., E. Kurashimo, H. Sato, N. Hirata, T. Iwasaki, and T. Kawanaka (2007), Interferometric seismic imaging of crustal structure using scattered teleseismic waves, Geophys. Res. Lett., 34, L19305, doi:10.1029/ 2007GL030633.

Bensen, G., M. Ritzwoller, M. Barmin, A. Levshin, F. Lin, M. Moschetti, N. Shapiro, and Y. Yang (2007), Processing seismic ambient noise data to obtain reliable broad-band surface wave dispersion measurements, Geophys. J. Int., 169, 1239-1260.
Chen, W.-P., M. Martin, T.-L. Tseng, R. Nowack, S.-H. Hung, and B.-S. Huang (2010), Shear-wave birefringence and current configuration of converging lithosphere under Tibet, Earth Planet. Sci. Lett., 295 297-304.

Claerbout, J. (1968), Synthesis of a layered medium from its acoustic transmission response, Geophysics, 33, 264-269.

Derode, A., E. Larose, M. Campillo, and M. Fink (2003), How to estimate the Green's function of a heterogeneous medium between two passive sensors? Application to acoustic waves, Appl. Phys. Lett., 83, 3054-3056.

Griffin, J., R. Nowack, W.-P. Chen, and T.-L. Tseng (2011), Velocity structure of the Tibetan lithosphere: Constraints from P-wave travel times of regional earhtquakes, Bull. Seismol. Soc. Am., 101, 1938-1947.

Hung, S.-H., W.-P. Chen, and L.-Y. Chiao (2011), A data-adaptive, multiscale approach of finite-frequency, traveltime tomography with special reference to $\mathrm{P}$ and $\mathrm{S}$ wave data from central Tibet, J. Geophys. Res., 116, B06307, doi:10.1029/2010JB008190.

Kennett, B., E. Engdahl, and R. Buland (1995), Constraints on seismic velocities in the Earth from travel times, Geophys. J. Int., 122, 108-124.

Langston, C. A. (1979), Structure under Mount Rainier, Washington, inferred from teleseismic body waves, J. Geophys. Res., 84, 4749-4762.

Nábělek, J., et al. (2009), Underplating in the Himalya-Tibet collision zone revealed by the Hi-CLIMB experiment, Science, 325, 1371-1374.

Nowack, R., S. Dasgupta, G. Schuster, and J.-M. Sheng (2006), Correlation migration using Gaussian beams of scattered teleseismic body waves, Bull. Seismol. Soc. Am., 96, 1-10.

Nowack, R., W.-P. Chen, and T.-L. Tseng (2010), Application of Gaussianbeam migration to multiscale imaging of the lithosphere beneath the Hi-CLIMB array in Tibet, Bull. Seismol. Soc. Am., 100, 1743-1754.

Randey, M., R. Tandukar, J. Avouac, J. Vergne, and T. Heritiér (1999), Seismotectonics of the Nepal Himalaya from a local seismic network, J. Asian Earth Sci., 17, 703-712.

Rost, S., and C. Thomas (2002), Array seismology: Methods and applications, Rev. Geophys., 40(3), 1008, doi:10.1029/2000RG000100.

Ruigrok, E., D. Draganov, and K. Wapenaar (2008), Global-scale seismic interferometry: Theory and numerical examples, Geophys. Prospect., $56,395-417$.

Ruigrok, E., X. Campman, D. Draganov, and K. Wapenaar (2010), Highresolution lithospheric imaging with seismic interferometry, Geophys. J. Int., 183, 339-357.

Schuster, G., J. Yu, J. Sheng, and J. Rickett (2004), Interferometric/daylight seismic imaging, Geophys. J. Int., 157, 838-852.

Snieder, R. (2004), Extracting the Green's function from the correlation of coda waves: A derivation based on stationary phase, Phys. Rev. E, 69 , 046610 .

Stein, S., and M. Wysession (2003), An Introduction to Seismology, Earthquakes, and Earth Structure, Blackwell, Malden, Mass.

Tseng, T.-L., W.-P. Chen, and R. L. Nowack (2009), Northward thinning of Tibetan crust revealed by virtual seismic profiles, Geophys. Res. Lett., 36 , L24304, doi:10.1029/2009GL040457.

Verschuur, D. (2006), Seismic Multiple Removal Techniques: Past, Present and Future, EAGE, Houten, Netherlands.

Wapenaar, K. (2003), Synthesis of an inhomogeneous medium from its acoustic transmission response, Geophysics, 68, 1756-1759.

Xu, Q., J. Zhao, S. Pei, and H. Liu (2011), The lithosphere-astenosphere boundary revealed by S-receiver functions from the Hi-CLIMB experiment, Geophys. J. Int., 187, 414-420.

Yilmaz, O., and S. Doherty (2000), Seismic Data Analysis: Processing, Inversion and Interpretation of Seismic Data, Soc. of Explor. Geophys., Tulsa, Okla.

Yin, A., and T. Harrison (2000), Geologic evolution of the HimalayanTibetan orogen, Annu. Rev. Earth Planet. Sci., 28, 211-280. 